\title{
Solar-wind control of plasma sheet dynamics
}

\author{
M. Myllys ${ }^{1}$, E. Kilpua ${ }^{1}$, and T. Pulkkinen ${ }^{2}$ \\ ${ }^{1}$ Department of Physics, University of Helsinki, Helsinki, P.O. Box 64, Finland \\ ${ }^{2}$ Aalto University School of Electrical Engineering, P.O. Box 1100, 00076 Aalto, Finland
}

Correspondence to: M. Myllys (minna.myllys@ helsinki.fi)

Received: 24 February 2015 - Revised: 21 May 2015 - Accepted: 22 May 2015 - Published: 21 July 2015

\begin{abstract}
The purpose of this study is to quantify how solarwind conditions affect the energy and plasma transport in the geomagnetic tail and its large-scale configuration. To identify the role of various effects, the magnetospheric data were sorted according to different solar-wind plasma and interplanetary magnetic field (IMF) parameters: speed, dynamic pressure, IMF north-south component, epsilon parameter, Auroral Electrojet (AE) index and IMF ultra low-frequency (ULF) fluctuation power. We study variations in the average flow speed pattern and the occurrence rate of fast flow bursts in the magnetotail during different solar-wind conditions using magnetospheric data from five Time History of Events and Macroscale Interactions during Substorms (THEMIS) mission spacecraft and solar-wind data from NASA's OMNIWeb. The time interval covers the years from 2008 to 2011 during the deep solar minimum between cycles 23 and 24 and the relatively quiet rising phase of cycle 24 . Hence, we investigate magnetospheric processes and solar-windmagnetospheric coupling during a relatively quiet state of the magnetosphere. We show that the occurrence rate of the fast $\left(\left|V_{\text {tail }}\right|>100 \mathrm{~km} \mathrm{~s}^{-1}\right)$ sunward flows varies under different solar-wind conditions more than the occurrence of the fast tailward flows. The occurrence frequency of the fast tailward flows does not change much with the solar-wind conditions. We also note that the sign of the IMF $B_{Z}$ has the most visible effect on the occurrence rate and pattern of the fast sunward flows. High-speed flow bursts are more common during the slow than fast solar-wind conditions.
\end{abstract}

Keywords. Magnetospheric physics (plasma sheet)

\section{Introduction}

The coupling of the solar wind to the magnetosphere has long been a subject of active research. In particular, the mechanisms of the energy transfer from the solar wind to the magnetosphere have been of great interest. According to the open model of the magnetosphere (Dungey, 1961), the magnetospheric magnetic field reconnects with the interplanetary magnetic field (IMF) on the dayside magnetopause. The opened geomagnetic field lines are transported to the magnetotail, where they are reconnected again. The resulting closed and stretched field lines migrate back to the dayside magnetopause. This motion generates the large-scale plasma convection in the magnetosphere.

The energy transfer efficiency from the solar wind to the Earth's magnetosphere is often studied by comparing various coupling functions (combinations of solar-wind parameters), as well as individual solar-wind parameters, with the geomagnetic indices (Meng et al., 1973; Stamper et al., 1999; Finch and Lockwood, 2007). For example, Akasofu's epsilon parameter (Perreault and Akasofu, 1978; Akasofu, 1981) is a commonly used coupling function to estimate the efficiency of the energy transfer to the inner magnetosphere. The dayside reconnection rate depends strongly on the sign of the IMF north-south component ( $B_{Z}$ component). In addition, the solar-wind speed has been found to correlate well with monthly and annual geomagnetic activity levels (Crooker et al., 1977; Finch et al., 2008).

Plasma sheet flows play a central role in the magnetospheric energy transport. The earliest studies focused mostly on the plasma sheet flow properties and studied the occurrence rate and spatial distributions of the fast and slow ion flows (Angelopoulos et al., 1992, 1993; Baumjohann et al., 1990; Shiokawa et al., 1997; Wang et al., 2006). Most of these studies defined the threshold of the high-speed flow 
to be $400 \mathrm{~km} \mathrm{~s}^{-1}$. The bursty bulk flow events (BBF) (Angelopoulos et al., 1992, 1993) are defined as periods when the flow speed average exceeds $100 \mathrm{~km} \mathrm{~s}^{-1}$ and is above $400 \mathrm{~km} \mathrm{~s}^{-1}$ (Baumjohann et al., 1990; Angelopoulos et al., 1992) for at least two samples during the 1 min period.

High-speed $\left(>250 \mathrm{~km} \mathrm{~s}^{-1}\right.$ ) flows have also been classified into two distinct categories based on their ion distributions: bulk flows and field-aligned beams (Nakamura et al., 1991; Raj et al., 2002). Bulk flows are perpendicular to the magnetic field at the neutral sheet but have a large field-aligned component at higher magnetic latitudes. Field-aligned beams are mainly detected away from the neutral sheet. The occurrence of BBF shows a dawn-dusk asymmetry in contrast to field-aligned beams (Raj et al., 2002).

In recent studies, the average bulk flow pattern and the characteristics of the plasma sheet flows with speed have been studied during the different IMF $B_{Z}$ conditions and substorm phases (Juusola et al., 2011a, b). However, there are only few studies of the large-scale magnetospheric flow pattern under different solar-wind conditions. One such study was recently published by Pulkkinen et al. (2013), who studied plasma sheet flows during steady magnetospheric convection events.

Previous studies of plasma sheet convection have shown that (1) the average ion bulk flow speed in the central plasma sheet has been found to be low, below $100 \mathrm{~km} \mathrm{~s}^{-1}$ (Baumjohann et al., 1990; Juusola et al., 2011b); (2) high-speed flows $\left(>400 \mathrm{~km} \mathrm{~s}^{-1}\right.$ ) occur in bursts mostly less than $10 \mathrm{~s}$ in duration (Baumjohann et al., 1990); (3) the slow flow pattern $\left(\left|v_{\perp}\right|<200 \mathrm{~km} \mathrm{~s}^{-1}\right)$ is not significantly different during northward and southward IMF conditions, but the overall flow speed is higher during southward IMF (Wang et al., 2006); (4) the largest occurrence rates of high-speed bursts $\left(>400 \mathrm{~km} \mathrm{~s}^{-1}\right)$ are found near the midnight meridian, and their occurrence peaks strongly in the sunward direction (Baumjohann et al., 1990); (5) high-speed bursts $\left(>400 \mathrm{~km} \mathrm{~s}^{-1}\right)$ are almost always directed earthward inside $-20 R_{\mathrm{E}}$ (Earth radius $=6372 \mathrm{~km}$ ), indicating that their source is beyond $X(\mathrm{GSM})<-19 R_{\mathrm{E}}$ (Shiokawa et al., 1997); (6) the occurrence rate of the high-speed burst decreases closer the Earth in the region from $X=-19$ to $-9 R_{\mathrm{E}}$ (Shiokawa et al., 1997); (7) the tailward BBFs are rare and infrequent but the ratio of tailward to earthward BBFs increases with distance from the Earth (Angelopoulus et al., 1994); (8) BBF events occur in only $10 \%$ of crossings (Angelopoulus et al., 1994).

Although on average the plasma flows are directed sunward in the plasma sheet, there are also tailward flows. The tailward flows can be classified depending on their $B_{Z}$ orientation. Tailward flows with southward $B_{Z}$ are rare and are usually considered to be caused by reconnection taking place earthward of the observation point. The tailward flows with northward $B_{Z}$ are often found in the region $-8>X>-15 R_{\mathrm{E}}\left(|Y|<5 R_{\mathrm{E}}\right)$, while most of the strong $\left(<-500 \mathrm{~km} \mathrm{~s}^{-1}\right)$ southward $B_{Z}$ flows occur in the range of
$-15>X>-30 R_{\mathrm{E}}$ (Ohtani et al., 2009). The dominance of tailward flows with northward $B_{Z}$ indicates that reconnection is not the primary cause of the flows. Ohtani et al. (2009) suggest that the sources of tailward flows could be the balloon instability and rebound of fast sunward flows near the Earth. The latter mechanism is supported by the fact that tailward flows are normally preceded by fast sunward flows and also by global Magnetohydrodynamics (MHD) simulations (Wiltberger et al., 2000).

The purpose of this study is to quantify how solar-wind conditions affect the energy and plasma transport in the geomagnetic tail. We use tail observations from the five THEMIS spacecraft during the years 2008-2011. During this period the THEMIS spacecraft spend a considerable time in the geomagnetic tail allowing us to compile statistical maps of plasma flow properties and to study the tail energy transport. In addition, this time period corresponds to the extended and prolonged solar activity minimum between solar cycle 23 and 24 and the relatively quiet rising phase of cycle 24. This allows us to investigate magnetospheric processes and solar-wind-magnetospheric coupling during a relatively quiet state of the magnetosphere. To uniquely identify the role of various effects, the magnetospheric data were sorted according to different solar-wind plasma parameters and by the Auroral Electrojet (AE) index. We study the variations in the average bulk flow speed pattern and the occurrence of the flow bursts exceeding 50 and $100 \mathrm{~km} \mathrm{~s}^{-1}$ and their preferred direction during different solar-wind conditions.

In this paper the first section describes the data sources and methods used in this study. The second section analyses the results. We end with discussion and conclusions.

\section{Data and methods}

\subsection{Data sources}

The solar wind and AE index data with 1 min resolution have been extracted from NASA Goddard Space Flight Center's OMNI data set through the OMNIWeb interface (http: //omniweb.gsfc.nasa.gov/). The data have been propagated to the nose of the Earth's bow shock. The parameters used are the solar-wind flow speed, IMF components in the GSM (geocentric solar magnetospheric coordinate system) coordinates, dynamic pressure and $\mathrm{AE}$ index.

The magnetospheric data come from the five THEMIS spacecraft. The THEMIS mission consists of five identically instrumented satellites orbiting the Earth at different distances. The orbits cover the magnetotail from 4 to $30 R_{\mathrm{E}}$. The ion velocity and density measurements come from the ESA instrument (McFadden et al., 2008) and the spin-averaged magnetic field measurements come from the Flux Gate Magnetometer (FGM) instrument (Auster et al., 2009). The data were interpolated with a 1 min time resolution and combined with the spacecraft position information. 


\subsection{Methods}

The magnetospheric data used to create the statistical maps were binned depending upon particular solar-wind or AE activity conditions. The median values of the distribution for each parameter were used to divide the magnetospheric data set into two parts to study the effect of each parameter on the magnetotail plasma flows and flow burst characteristics. The limit (median) values and the used solar-wind parameters are also listed in Table 1. A few exceptions were made to using the median values: for the IMF $Z$ component, we used the limit value of $0 \mathrm{nT}$. The median value for the $\mathrm{AE}$ index (60 nT) does not delimit geomagnetically quiet and active times, but the data coverage turned out to be poor when we tried to use the upper quartile of the $\mathrm{AE}$ index distribution.

A $1 \mathrm{~h}$ sliding average time series for each solar-wind parameter was computed preceding the tail measurement. In the case of the AE index, the $1 \mathrm{~h}$ sliding average time series was computed following the tail measurement. We chose a $1 \mathrm{~h}$ average since it roughly corresponds to the time it takes for the solar-wind plasma to be transported to the magnetotail. Time steps were connected directly with the THEMIS observation at the same time. The average plasma sheet flow in the GSM $X Y$ plane were computed using the THEMIS ion $V_{X}$ and $V_{Y}$ velocity components $\left(V_{\text {tail }}^{2}=V_{X}^{2}+V_{Y}^{2}\right)$. We excluded the $Z$ component of the velocity because the component mainly consists of the vertical movement of the plasma sheet and is not part of the plasma convection. The flow speed was multiplied by the sign of the flow $V_{X}$ component in order to differentiate predominantly sunward $\left(V_{X}>0\right)$ and tailward $\left(V_{X}<0\right)$ flows.

We cover the region $-30<X<0 R_{\mathrm{E}}$ and $-10<Y<10 R_{\mathrm{E}}$, where $X$ and $Y$ are coordinates in the GSM coordinate system. THEMIS observations were removed if the $|Z|$ component was greater than $5 R_{\mathrm{E}}$. To distinguish the plasma sheet periods from the lobe periods, we used the criterion ion $\beta>0.5$ (Raj et al., 2002; Juusola et al., 2011b) for the plasma sheet. The velocity vectors contain both the perpendicular and parallel components. The magnetospheric data set may include some field-aligned beam samples, but according to Raj et al. (2002) the criterion $\beta>0.5$ should remove $45 \%$ of these undesired high-speed $\left(>250 \mathrm{~km} \mathrm{~s}^{-1}\right.$ ) samples. Even if the results are shown in the area $-30<X<0 R_{\mathrm{E}}$, we note that the data quality may decrease when $X>-10 R_{\mathrm{E}}$ because the rising temperatures may cause a significant part of the ion distribution functions to be above the high-energy threshold of the ESA ion instruments.

The magnetotail region was divided into $1 R_{\mathrm{E}} \times 1 R_{\mathrm{E}}$ bins and the average of the observations inside each bin were calculated. To smooth the data and increase the statistics, the bin size was increased by taking observations from the bin center to $\pm 1 R_{\mathrm{E}}$, both in $X$ and $Y$ directions, resulting in an average bin size of $2 \times 2 R_{\mathrm{E}}^{2}$. At least 25 measurement points were required for each $2 \times 2 R_{\mathrm{E}}^{2}$ bin when the flow averages
Table 1. Solar-wind parameters and their limit values.

\begin{tabular}{ll}
\hline Parameter (unit) & Limit \\
\hline Solar-wind speed, $V\left(\mathrm{~km} \mathrm{~s}^{-1}\right)$ & 400 \\
Dynamic pressure, $P_{\text {dyn }}(\mathrm{nPa})$ & 1.4 \\
IMF $Z$ component, $B_{Z},(\mathrm{nT})$ & 0 \\
$B_{Z}$ ULF power $\left(\mathrm{nT}^{2} \mathrm{~min}^{-1}\right)$ & 0.26 \\
$\mathrm{AE}$ index (nT) & 60 \\
Epsilon, $\epsilon(\mathrm{W})$ & $5 \times 10^{10}$ \\
\hline
\end{tabular}

and the occurrence rate of the high-speed bursts were computed. The flow averages were calculated separately for flow speeds $<50 \mathrm{~km} \mathrm{~s}^{-1}$ (convection-related effects) and for flow speeds $>50 \mathrm{~km} \mathrm{~s}^{-1}$ (bursts created by dynamic events in the tail). Previous studies (Baumjohann et al., 1990) have shown that the mean central plasma sheet velocity is typically below $50 \mathrm{~km} \mathrm{~s}^{-1}$. Hence, we decided to choose $50 \mathrm{~km} \mathrm{~s}^{-1}$ as the limit value. Because we use 1 min averages, our plasma flow values are lower than in previous studies. Consequently our flow amplitudes cannot be directly compared with the previous results examining data at higher time resolution. We have divided all the measurement points into four bins according to speed and direction to study the properties of each velocity bin separately: $V_{\text {tail }}<-50 \mathrm{~km} \mathrm{~s}^{-1} ;-50 \mathrm{~km} \mathrm{~s}^{-1}<V_{\text {tail }}<0$; $0<V_{\text {tail }}<50 \mathrm{~km} \mathrm{~s}^{-1}$ and $V_{\text {tail }}>50 \mathrm{~km} \mathrm{~s}^{-1}$.

\section{Results}

\subsection{Plasma sheet flow, all data}

We first investigate plasma flow patterns for all data during 2008-2011 and then proceed to separate the data according to solar-wind and geomagnetic conditions. Figures 1 and 2 show the averages of the plasma flow speed for sunward (right panels) and tailward (left panels) flows of less than $50 \mathrm{~km} \mathrm{~s}^{-1}$ and greater than $50 \mathrm{~km} \mathrm{~s}^{-1}$. The flows have been divided according to the sign of the $V_{X}$ component. The left panels show the sunward flows and the right panels the tailward flows.

Figure 3 shows the occurrence rate of fast flow bursts. Since flow bursts slow down considerably when they approach the Earth, we have used a different threshold for flow bursts in the regions $-20<X<0 R_{\mathrm{E}}$ and $-30<X<-10 R_{\mathrm{E}}$ (separated by a small gap in the figure). In the near-Earth tail the threshold for a flow burst is $50 \mathrm{~km} \mathrm{~s}^{-1}$, while in the mid-tail we used the limit $100 \mathrm{~km} \mathrm{~s}^{-1}$.

The Fig. 3 shows that the occurrence rate of fast sunward bursts is much higher than the rate of tailward bursts. Tailward flow bursts with speeds exceeding $100 \mathrm{~km} \mathrm{~s}^{-1}$ are rare, and the figure shows that they mostly exist when $X \lesssim-20 R_{\mathrm{E}}$. The occurrence rate of fast sunward bursts rises with the increasing distance from the Earth. A notable increase occurs when $X \approx-15 R_{\mathrm{E}}$, which is a few $R_{\mathrm{E}}$ earlier than the 


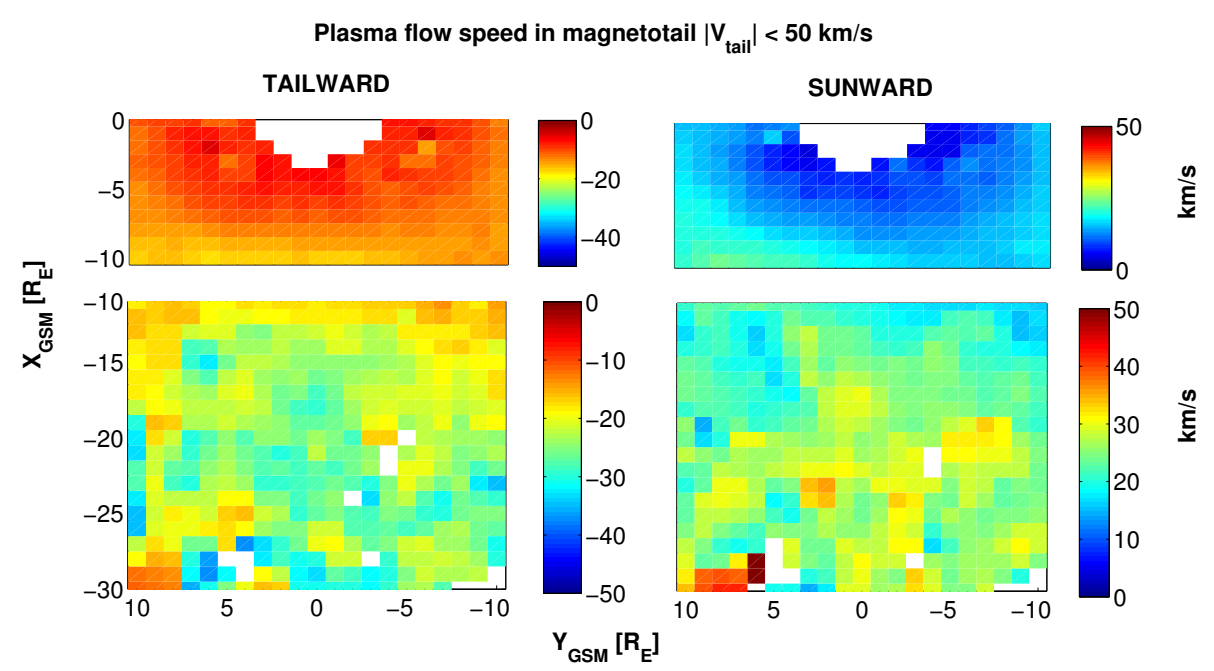

Figure 1. Average plasma flow pattern for slows flows $\left(\left|V_{\text {tail }}\right|<50 \mathrm{~km} \mathrm{~s}^{-1}\right)$ in 2008-2011. The colors show the average speed of the bin.

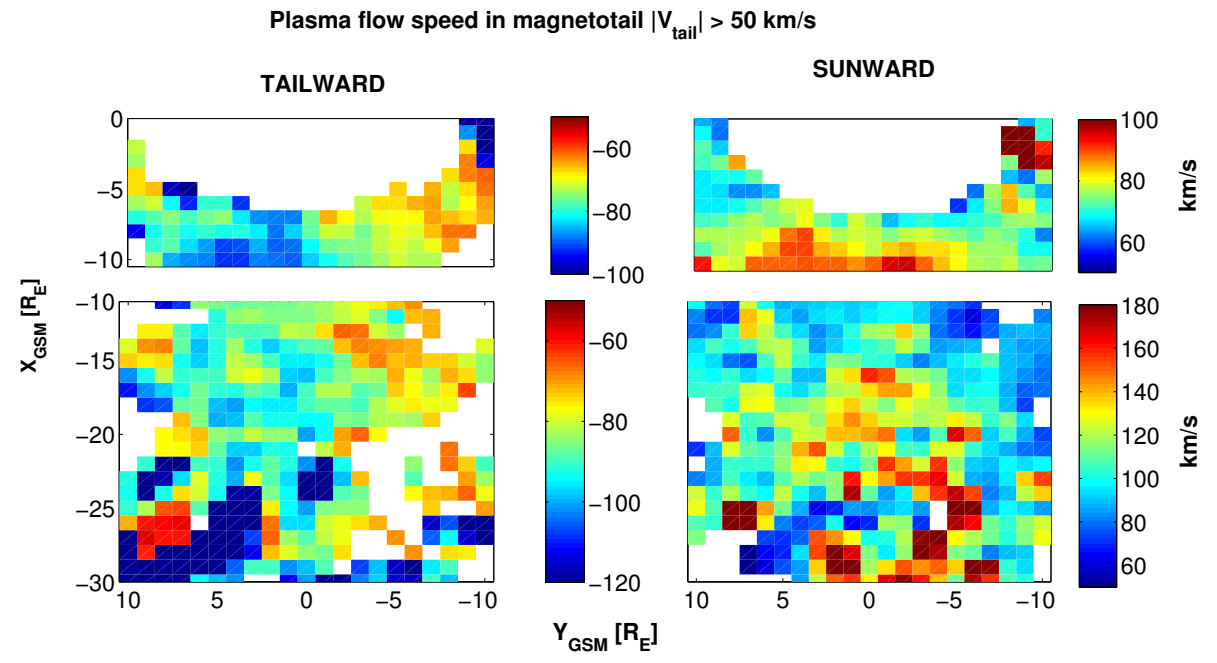

Figure 2. Average plasma flow pattern for fast flows $\left(\left|V_{\text {tail }}\right|>50 \mathrm{~km} \mathrm{~s}^{-1}\right)$ in $2008-2011$. The colors show the average speed of the bin.

starting point of the majority of tailward flows. In the case of bursts $>50 \mathrm{~km} \mathrm{~s}^{-1}$, the difference between the occurrence of the sunward and tailward bursts is not as clear as in the case bursts $>100 \mathrm{~km} \mathrm{~s}^{-1}$, but it is still visible. The bursts $>|50| \mathrm{km} \mathrm{s}^{-1}$ seem to cease when $X>-10 R_{\mathrm{E}}$. The lack of measurement points (Fig. 5) for both fast (Fig. 4) and slow flows (not shown here) on the dawnside when $X$ is between -20 to $-30 R_{\mathrm{E}}$ may be the reason for the high occurrence rate of fast bursts inside the region (Fig. 3).

The average plasma flow maps $\left(\left|V_{\text {tail }}\right|>50 \mathrm{~km} \mathrm{~s}^{-1}\right.$, Fig. 2) show a dawn-dusk asymmetry of the sunward and tailward flow speed. The clear asymmetry of the flows is probably due to the asymmetry of the observations (Figs. 4 and 5). Such an asymmetry is not visible in the case of small flows $\left(\left|V_{\text {tail }}\right|<50 \mathrm{~km} \mathrm{~s}^{-1}\right.$, Fig. 1). The local maximum of the occurrence rate of tailward flows at $X \approx-20-15 R_{\mathrm{E}}$ (Fig. 4) may be due to the sporadic appearance of a near-Earth neutral line (NENL).

\subsection{Average plasma sheet flow during different solar-wind conditions}

Next, we present statistical maps of the average flow speed for sunward and tailward flows and the occurrence of flow bursts organized with the selected solar-wind parameters (and derived coupling functions) and the geomagnetic activity level. Statistical maps (Figs. 6, 7 and 8) show common features regardless of the solar-wind and geomagnetic activity conditions: (1) fast (> 50 and $>100 \mathrm{~km} \mathrm{~s}^{-1}$ ) sunward bursts are more common than tailward bursts (the bottom panels in Figs. 6, 7 and 8); (2) dawn-dusk asymmetry of the sunward flows is $>100 \mathrm{~km} \mathrm{~s}^{-1}$; (3) there is an increase in the occurrence rate of fast bursts $\left(\left|V_{\text {tail }}\right|>100 \mathrm{~km} \mathrm{~s}^{-1}\right)$ when 


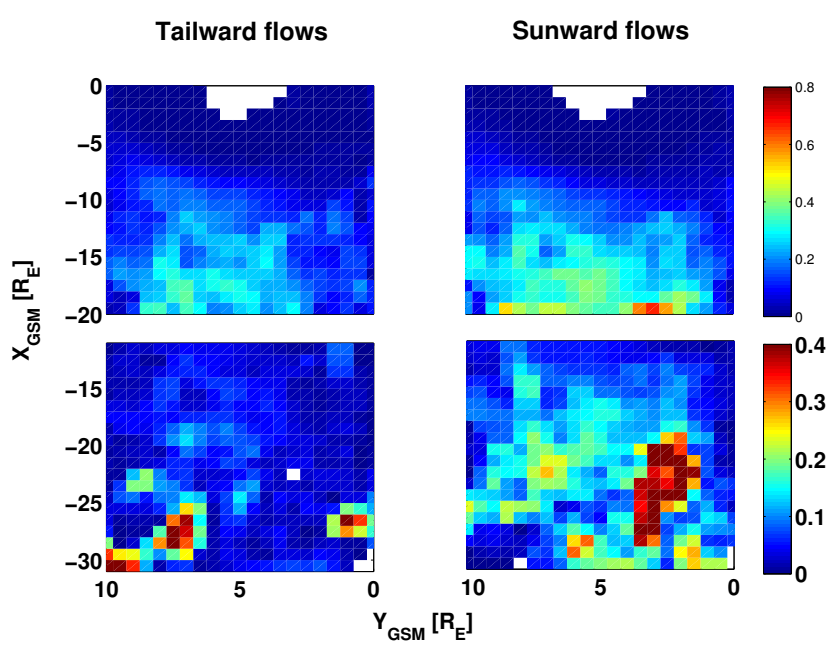

Figure 3. Percentage of observations during which $1 \mathrm{~min}$ averaged flow speed exceeds $50 \mathrm{~km} \mathrm{~s}^{-1}$ (upper panels) or $100 \mathrm{~km} \mathrm{~s}^{-1}$ (lower panels) during studied time interval. The data set is divided into two groups based on the sign of the $V_{X}$ component.

$X<-20 R_{\mathrm{E}}$; (4) the occurrence rate of the tailward flow bursts over $100 \mathrm{~km} \mathrm{~s}^{-1}$ does not significantly vary with the solar wind conditions; (5) the average slow flow pattern is not affected by the solar-wind conditions.

The occurrence maps showing the high-speed bursts (the bottom panels in Figs. 6, 7 and 8) demonstrate that the most visible changes in the flow pattern during different solarwind conditions are caused by the decrease in fast sunward flows rather than by a significant increase in tailward bursts. The flow pattern of the tailward bursts seems to be steadier than the pattern of sunward flows.

\subsubsection{Solar-wind speed}

The top and middle right-hand panels of Fig. 6 show the average tail plasma flow for sunward and tailward flows when $\left|V_{\text {tail }}\right|<50 \mathrm{~km} \mathrm{~s}^{-1}$ and $\left|V_{\text {tail }}\right|>50 \mathrm{~km} \mathrm{~s}^{-1}$, respectively, using the division according to the solar-wind speed (see Table 1).

The occurrence rates of tailward and sunward flow bursts which exceed $50 \mathrm{~km} \mathrm{~s}^{-1}\left(X>-20 R_{\mathrm{E}}\right)$ and $100 \mathrm{~km} \mathrm{~s}^{-1}$ $(X<-10)$ during the high and slow solar-wind periods (the bottom-right panels) are shown in Fig. 6. It can be seen that the occurrence rate of sunward bursts exceeding $100 \mathrm{~km} \mathrm{~s}^{-1}$ varies significantly with changing solar-wind speed conditions. The occurrence rate is much higher during the slow wind than during the fast wind. The high occurrence rate region is also more concentrated on the dusk side during the slower solar wind compared to fast solar-wind times. The number of fast bursts decreases drastically when $X>-20 R_{\mathrm{E}}$ during the slower solar wind, while for the faster wind the decrease starts already at $X>-15 R_{\mathrm{E}}$. The occurrence range of the tailward bursts are not appreciably affected by the solar- wind speed but the tailward bursts exceeding $100 \mathrm{~km} \mathrm{~s}^{-1}$ are a bit more common between $-15>X>-20 R_{\mathrm{E}}$.

\subsubsection{Solar-wind dynamic pressure}

The left-hand top and middle panels of Fig. 6 show the averages of fast $\left(\left|V_{\text {taii }}\right|>50 \mathrm{~km} \mathrm{~s}^{-1}\right)$ and slow $\left(\left|V_{\text {tail }}\right|<50 \mathrm{~km} \mathrm{~s}^{-1}\right)$ tail plasma flows during two different solar-wind dynamic pressure levels (see limiting value from Table 1). During low $P_{\mathrm{dyn}}$, the average fast plasma sheet flows are higher at the midnight meridian when $-20<X<-15 R_{\mathrm{E}}$.

The bottom-left panels of Fig. 6 show the occurrence rate of fast $\left(\left|V_{\text {tail }}\right|>50 \mathrm{~km} \mathrm{~s}^{-1}\right.$ and $\left.\left|V_{\text {tail }}\right|>100 \mathrm{~km} \mathrm{~s}^{-1}\right)$ sunward and tailward bursts during the same pressure conditions. It can be seen from the figures that the occurrence rate of highspeed bursts increases during high solar-wind pressure conditions. Especially inside the area $-20<X<-15 R_{\mathrm{E}}$ the sunward bursts exceeding 100 and $50 \mathrm{~km} \mathrm{~s}^{-1}$ have a wider occurrence range during high- $P_{\mathrm{dyn}}$ conditions than during the low- $P_{\text {dyn }}$ conditions. During the low-pressure periods the fast flows are clearly focused on the narrow channel at the midnight meridian. The occurrence range of fast tailward bursts is slightly expanded during higher- $P_{\text {dyn }}$ periods. In the case of tailward bursts, the bursts exceeding $50 \mathrm{~km} \mathrm{~s}^{-1}$ occur more often between $X>-15$ and $X<-10 R_{\mathrm{E}}$ than during lower$P_{\text {dyn }}$ conditions.

\subsubsection{Interplanetary magnetic field direction}

The bottom-right panels of Fig. 7 show the occurrence rate of high-speed tail and sunward bursts during the southward and northward IMF component conditions. In turn, Fig. 7 (righthand top and middle panels) shows the net plasma flow during the same $B_{Z}$ criteria for sunward and tailward flows with $\left|V_{\text {tail }}\right|>50 \mathrm{~km} \mathrm{~s}^{-1}$ and $\left|V_{\text {tail }}\right|<50 \mathrm{~km} \mathrm{~s}^{-1}$.

It can be seen that IMF $B_{Z}$ has a clear effect on the occurrence rate of high-speed bursts. In particular, $B_{Z}$ seems to have the largest effect on the occurrence rate of fast tailward bursts $\left(\left|V_{\text {tail }}\right|>50\right.$ and $\left.100 \mathrm{~km} \mathrm{~s}^{-1}\right)$ among all investigated solar-wind parameters. The number of $>50 \mathrm{~km} \mathrm{~s}^{-1}$ tailward bursts increases significantly during negative (southern) $B_{Z}$ conditions. Also, the number of sunward bursts increases when $B_{Z}<0$, and the bursts are spread over a larger area. In turn, for northward IMF conditions, the sunward bursts are focused inside a narrow channel near the magnetic midnight.

The sunward net flow maps (Fig. 7, the middle righthand panels) show that during periods in which $B_{Z}<0 \mathrm{nT}$ the average sunward plasma flow intensifies in the region $-20<X<-12 R_{\mathrm{E}}$ near the midnight meridian. The sunward net flow is also intensified when $X<-20 R_{\mathrm{E}}$, and the fast flows are spread into a wider region than during the northward $B_{Z}$. However, the poorer data coverage during the northward $B_{Z}$ limits the observations. There is an area at the midnight meridian when $X \approx-20 R_{\mathrm{E}}$ which lacks fast $\left(>100 \mathrm{~km} \mathrm{~s}^{-1}\right.$ ) sunward bursts compared to the other bins 
Plasma flow speed in magnetotail $\left|\mathrm{V}_{\text {tail }}\right|>50 \mathrm{~km} / \mathrm{s}$

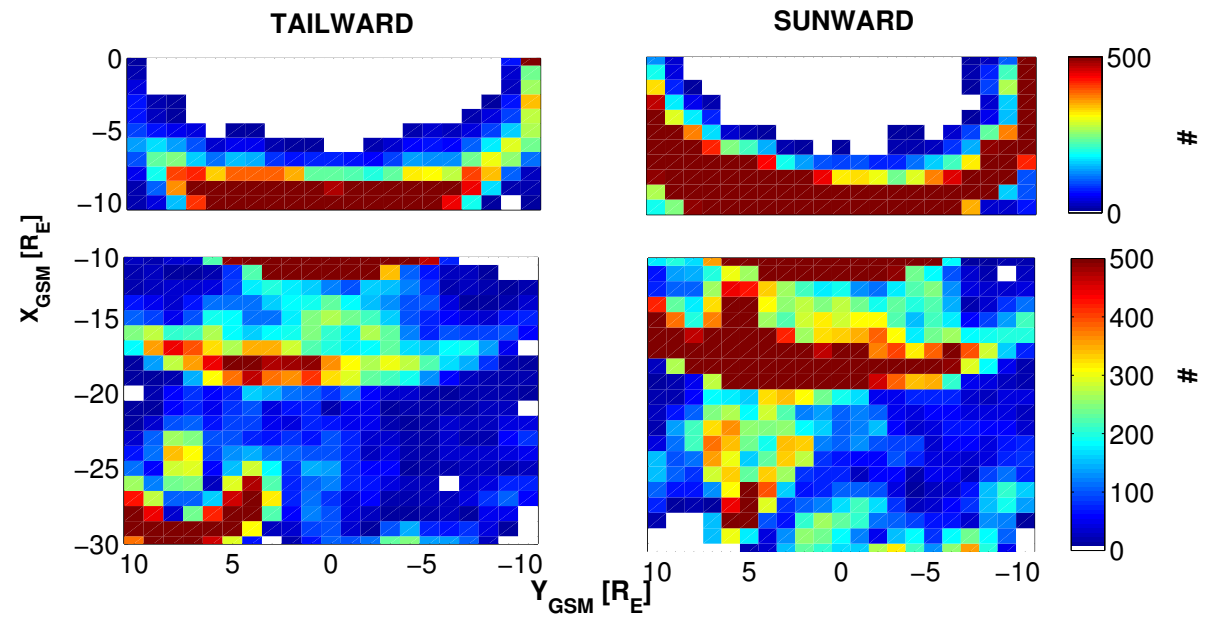

Figure 4. Number of 1 min averaged data points $\left(\left|V_{\text {tail }}\right|>50 \mathrm{~km} \mathrm{~s}^{-1}\right)$ in 2008-2011.

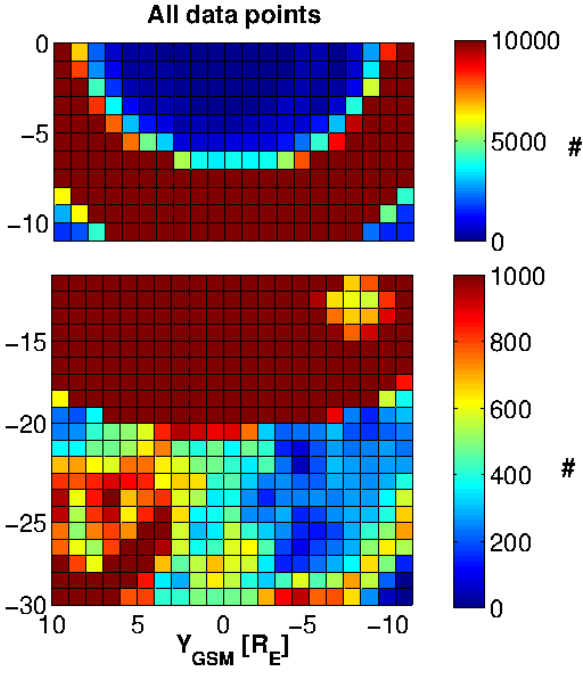

Figure 5. Number of all 1 min averaged data points in 2008-2011.

around it. This area also features weaker net plasma flow in the maps of an average flow speed of $>50 \mathrm{~km} \mathrm{~s}^{-1}$. The weakening of the sunward flows in the area may be related to the formation of the reconnection line. The tailward net flow does not show large changes during different $B_{Z}$ conditions, but this may also be because of the limited data set during the positive $B_{Z}$. Instead, the tailward net flow pattern for slow flows $\left(\left|V_{\text {tail }}\right|<50 \mathrm{~km} \mathrm{~s}^{-1}\right)$ shows clear differences during different IMF $B_{Z}$ times (Fig. 7, the top-right panels). The average flow is slower when $B_{Z}$ is positive.

\subsubsection{Ultra low-frequency power}

We also investigated the effect of ultra low-frequency (ULF) $B_{Z}$ power on the tail plasma flow pattern and burst occurrence. The 2-12 min frequency range was used to calculate the ULF powers. The results are presented in the left panels of Fig. 7.

The convection pattern stayed almost constant regardless of the ULF $B_{Z}$ power level. The data coverage is poor, but according to the existing data, the sunward flow $\left(\left|V_{\text {tail }}\right|>50 \mathrm{~km} \mathrm{~s}^{-1}\right)$ is slightly intensified in the range of $-20<X<-10 R_{\mathrm{E}}$ during the high ULF power conditions, but otherwise the flow patterns remain relatively similar. The occurrence rate of tailward $\left(>50\right.$ and $>100 \mathrm{~km} \mathrm{~s}^{-1}$ ) bursts does not show any significant difference between the two ULF $B_{Z}$ power levels. In the case of sunward bursts, the only visible difference occurs when $-20<X<-15 R_{\mathrm{E}}$.

\subsubsection{AE index}

The magnetospheric flow pattern and the occurrence rate of fast flow bursts have also been studied during two different $\mathrm{AE}$ index levels. The results are shown in Fig. 8. AE describes the geomagnetic disturbance level of the auroral zone; thus, it combines the magnetospheric flows to ground observations. AE activity increases the number of sunward and tailward high-speed bursts. Especially the flow bursts exceeding $50 \mathrm{~km} \mathrm{~s}^{-1}$ are increased in the mid-meridians during higher AE activity. The maps which show the mean net flow of fast flows (Fig. 8, the middle right-hand panels) are reminiscent of the average flow maps during different solarwind speed conditions, especially when $-10<X<-20 R_{\mathrm{E}}$. The slow flow pattern $\left(<50 \mathrm{~km} \mathrm{~s}^{-1}\right)$ does not seem to be connected with the AE activity (Fig. 8, the top-right panels). 

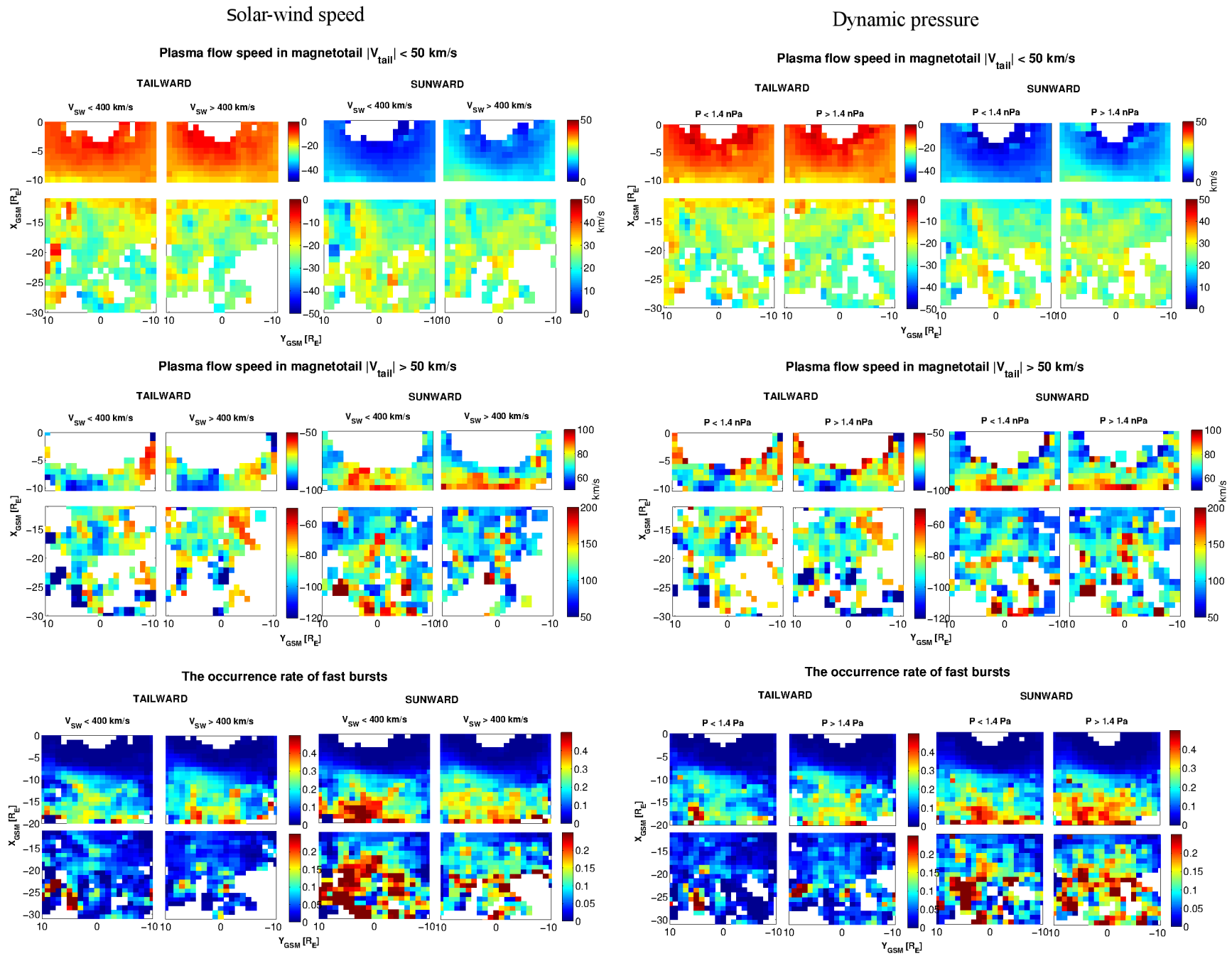

Figure 6. Average plasma flow pattern for slow ( $\left|V_{\text {tail }}\right|<50 \mathrm{~km} \mathrm{~s}^{-1}$, top panels) and fast flows $\left(\left|V_{\text {tail }}\right|>50 \mathrm{~km} \mathrm{~s}^{-1}\right.$, middle panels), ratio between the time steps when tail flow bursts exceeded $50 \mathrm{~km} \mathrm{~s}^{-1}$ (bottom panels, region $0>X>-20 R_{\mathrm{E}}$ ) or $100 \mathrm{~km} \mathrm{~s}{ }^{-1}$ (bottom panels, region $-20>X>-30 R_{\mathrm{E}}$ ), and all time steps when the data points are divided into groups based on the solar-wind speed and dynamic pressure. The limit for the $1 \mathrm{~h}$ sliding average solar-wind speed and pressure values are written above the maps. The tailward and sunward flows are shown separately.

\subsubsection{Epsilon parameter}

One coupling function was chosen for this study: Akasofu's epsilon parameter which is given in SI units as

$\epsilon=\frac{4 \pi}{\mu_{0}} V B^{2} \sin ^{4}\left(\frac{\theta}{2}\right) l_{0}^{2}$.

In the formula, $l_{0}$ is an empirically determined scale length, set to $7 R_{\mathrm{E}}, B$ is the magnitude of the IMF, $V$ is the solar-wind speed, and $\theta$ is the "clock angle" between the $Z$ axis and the projection of the IMF onto the $Y Z$ plane in the GSM coordinates. The epsilon parameter is usually used to estimate the energy transfer to the inner magnetosphere. This parameter depends on the interplanetary magnetic field
$(B)$ and the solar-wind speed $(V)$. When the $1 \mathrm{~h}$ mean epsilon parameter is $>5 \times 10^{10} \mathrm{~W}$, the sunward flow is clearly increased in the region $-25<X<-10 R_{\mathrm{E}}$ (Fig. 8, the middle left-hand panels). As can be seen in Fig. 8, the occurrence rates of sunward and tailward bursts do not vary significantly with the amplitude of the epsilon parameter. The results may be affected by the fact that the limit value used for epsilon does not clearly represent high or low epsilon activity. During high-epsilon periods the highest occurrence rates of flow bursts are concentrated near the midnight meridian when $X<-20 R_{\mathrm{E}}$, in contrast with the low-epsilon periods when there is a lack of high bursts in mid-meridians. 

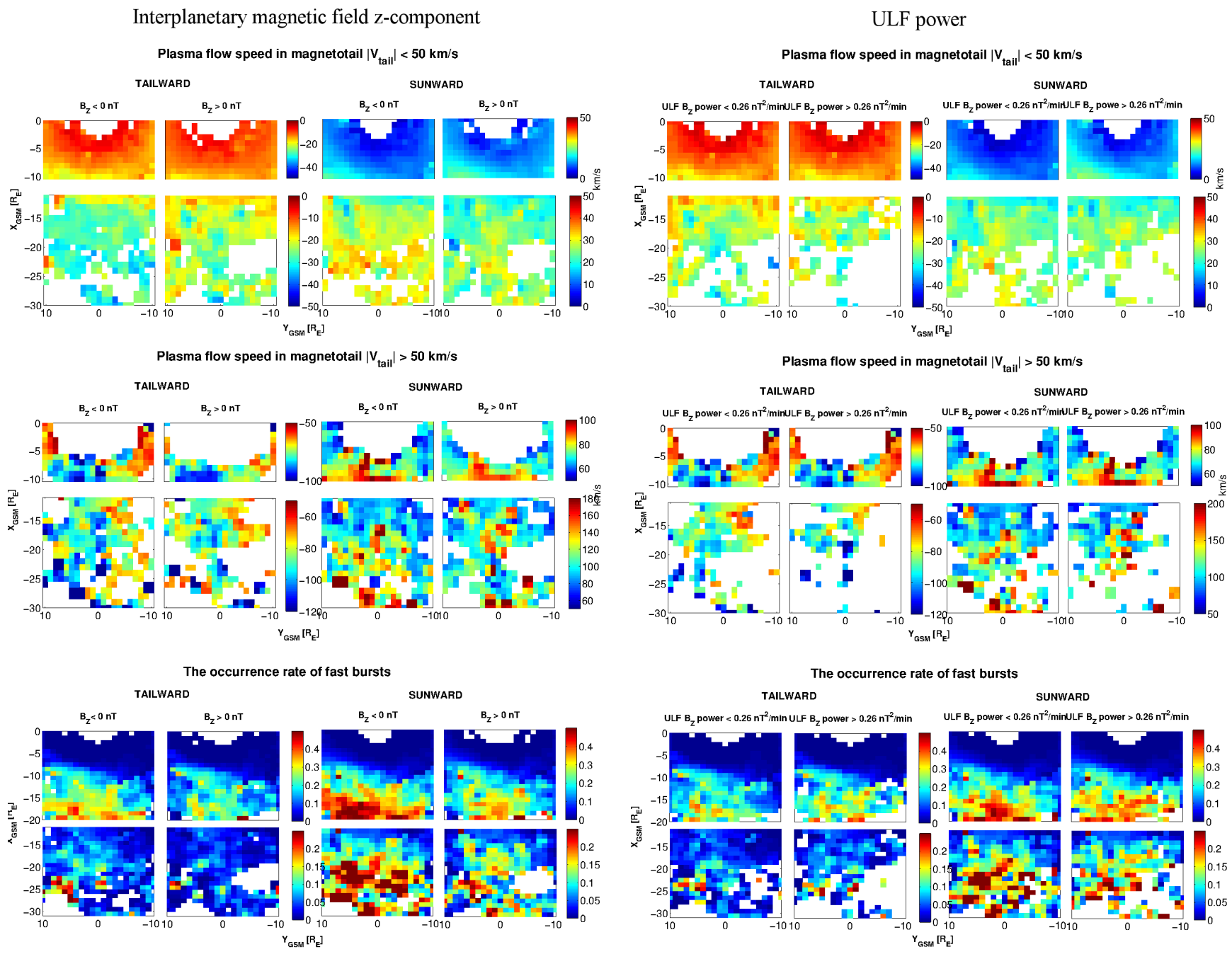

Figure 7. Average plasma flow pattern for slow ( $\left|V_{\text {tail }}\right|<50 \mathrm{~km} \mathrm{~s}^{-1}$, top panels) and fast flows $\left(\left|V_{\text {tail }}\right|>50 \mathrm{~km} \mathrm{~s}^{-1}\right.$, middle panels), ratio between the time steps when tail flow bursts exceeded $50 \mathrm{~km} \mathrm{~s}^{-1}$ (bottom panels, region $0>X>-20 R_{\mathrm{E}}$ ) or $100 \mathrm{~km} \mathrm{~s}{ }^{-1}$ (bottom panels, region $-20>X>-30 R_{\mathrm{E}}$ ), and all time steps when the data points are divided into groups based on the interplanetary magnetic field $z$ component and ULF $B_{Z}$ power. The limit for the $1 \mathrm{~h}$ sliding average $B_{Z}$ and ULF power values are written above the maps. The tailward and sunward flows are shown separately.

\section{Discussion and conclusions}

We have studied the large-scale plasma transport in the magnetotail during different solar-wind conditions in 2008-2011. To find out the role of different solar-wind parameters and their levels in relation to the average sunward and tailward plasma flows and the occurrence rate of fast plasma bursts, the magnetospheric data were sorted according to the solarwind measurements. The results are shown in statistical maps (Figs. 6, 7, and 8). This study highlights the active energy flow in the plasma sheet in the primarily weakly driven magnetosphere.

The statistical maps confirm some of the features that have been observed in earlier studies. Most of the time the plasma flow is less than $100 \mathrm{~km} \mathrm{~s}^{-1}$, as can be seen in Fig. 3 as well as in the previously published studies (Angelopoulus et al., 1994; Juusola et al., 2011b). The result showing that the occurrence rate of fast sunward bursts increases with increasing distance from the Earth is in agreement with earlier studies (Shiokawa et al., 1997; Juusola et al., 2011b). The tailward fast bursts were known to be rare (Ohtani et al., 2009), which was also observed in this study. According to Baumjohann et al. (1990), high-speed (> $400 \mathrm{~km} \mathrm{~s}^{-1}$ ) bursts are more frequent during geomagnetically disturbed times (high $\mathrm{AE}$ ) but also occur during low auroral electrojet index conditions. The statistical maps which have been formed based on the different $\mathrm{AE}$ index levels show an increase in the occurrence rate of the high-speed bursts during higher AE times. Shiokawa et al. (1997) found that the flow bursts $\left(>400 \mathrm{~km} \mathrm{~s}^{-1}\right.$ ) are mostly directed earthward when $X>-20 R_{\mathrm{E}}$. According 

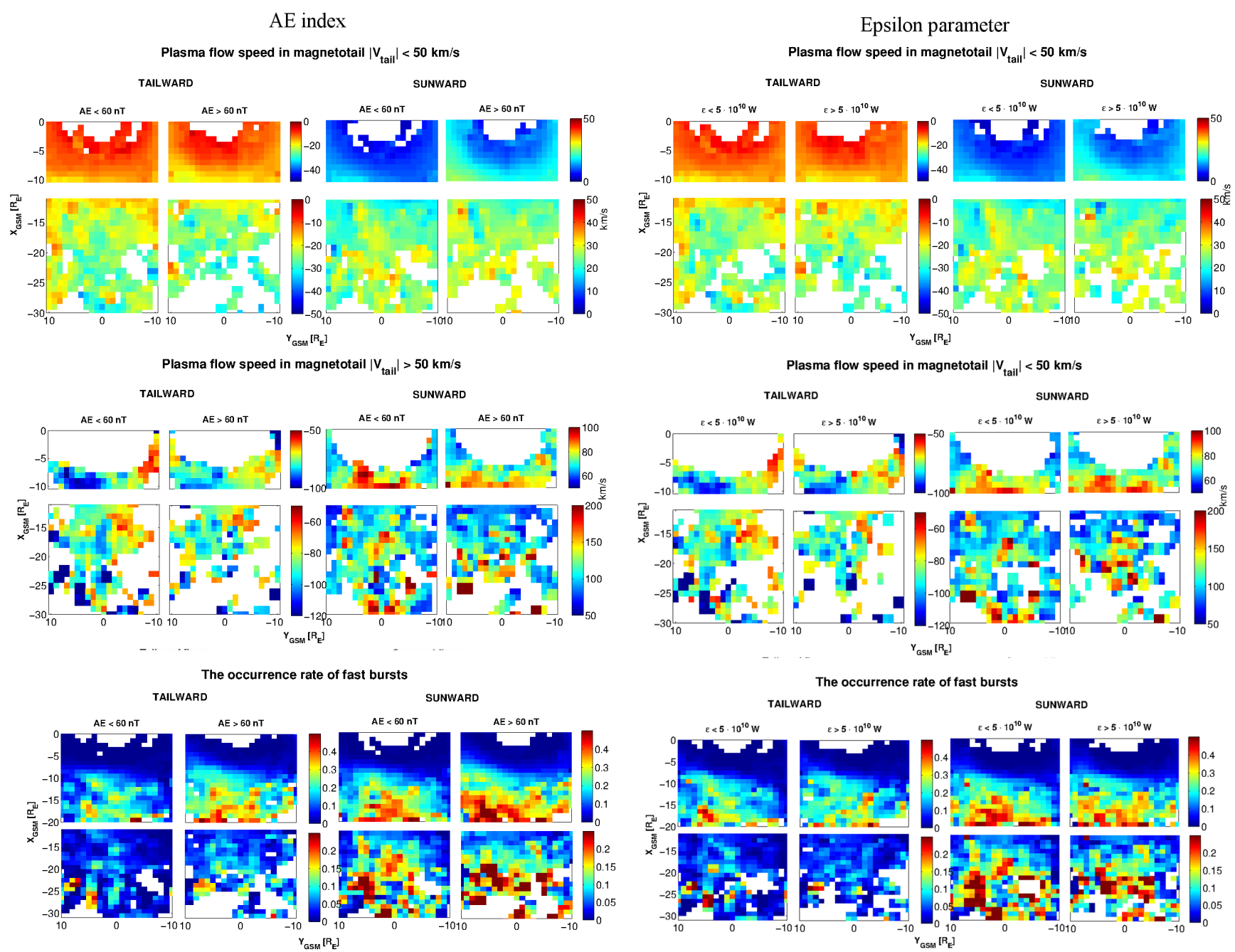

Figure 8. Average plasma flow pattern for slow $\left(\left|V_{\text {tail }}\right|<50 \mathrm{~km} \mathrm{~s}^{-1}\right.$, top panels) and fast flows $\left(\left|V_{\text {tail }}\right|>50 \mathrm{~km} \mathrm{~s}^{-1}\right.$, middle panels), ratio between the time steps when tail flow bursts exceeded $50 \mathrm{~km} \mathrm{~s}^{-1}$ (bottom panels, region $0>X>-20 R_{\mathrm{E}}$ ) or $100 \mathrm{~km} \mathrm{~s}^{-1}$ (bottom panels, region $-20>X>-30 R_{\mathrm{E}}$ ), and all time steps when the data points are divided into groups based on the AE index and epsilon parameter. The limit for the $1 \mathrm{~h}$ sliding average AE index and epsilon parameter values are written above the maps. The tailward and sunward flows are shown separately.

to the occurrence maps of fast bursts (Figs. 6, 7 and 8, the bottom panels), the tailward bursts $>100 \mathrm{~km} \mathrm{~s}^{-1}$ are mainly located beyond $-20 R_{\mathrm{E}}$ regardless of the solar-wind conditions.

Variations in the plasma flow pattern in the magnetotail during the northern and southern IMF periods are likely to reflect differences in the energy transfer from the solar wind to the magnetosphere by viscous interaction (Tsurutani and Gonzalez, 1995) and reconnection processes (Dungey, 1961). The efficiency of solar-wind energy injection into the magnetosphere is less efficient via viscous interaction than reconnection (Tsurutani and Gonzalez, 1995), which can be seen in this study as a weaker net plasma flow in the magnetotail in the northward IMF in Fig. 7.
Ohtani et al. (2009) has stated that reconnection cannot be the primary cause for the tailward flows in the near-Earth tail $\left(-8>X>-15 R_{\mathrm{E}}\right.$ and $\left.|Y|<5 R_{\mathrm{E}}\right)$. He also noted that changes in the external conditions (IMF orientation and dynamic pressure) in terms of direct momentum transport from the solar wind to the magnetosphere (vortex motion caused by Kelvin-Helmholtz instability) cannot fully explain the flows. He suggested that the cause of fast tailward flows must be internal and local. Based on this study the occurrence rate of tailward-directed flows remains very similar regardless of the solar-wind conditions, unlike the occurrence of sunward bursts. The sunward flow bursts display a clear change, for example, with the IMF direction and the speed of solar wind. This suggests that the tailward flows are not directly dependent on the solar-wind parameters. 
Figure 2, which includes all the 4-year data, reveals an area of fast tailward flows at midnight meridian when $-25 R_{\mathrm{E}}<X<-20 R_{\mathrm{E}}$. The previous studies have estimated the location of NENL to be at $-20 R_{\mathrm{E}}<X_{\mathrm{GSM}}<-30 R_{\mathrm{E}}$ (Nagai et al., 1998). The tailward net flow when $X<-20 R_{\mathrm{E}}$ is likely caused by the formation of an NENL. The location of NENL is also visible in some of the average plasma flow maps (Figs. 6, 7 and 8), which are sorted based on the solar-wind plasma parameters. For example, the southern $B_{Z}$, the upper limit of epsilon, dynamic pressure and solarwind speed also show the NENL when $X \approx-20 R_{\mathrm{E}}$.

In summary, we found that the occurrence rate of the fast $\left(\left|V_{\text {tail }}\right|>100 \mathrm{~km} \mathrm{~s}^{-1}\right)$ sunward flows varies in different solarwind conditions more than the occurrence of the fast tailward flows. In general, the number of fast tailward flows does not change much with the solar wind. We also noted that the sign of the IMF $B_{z}$ has the most visible effect on the occurrence rate and pattern of fast sunward flows. The flow bursts exceeding 50 and $100 \mathrm{~km} \mathrm{~s}^{-1}$ are also more common during slow wind than high solar wind; to our knowledge, these bursts have not been reported previously. This result still needs further investigations; in particular, the effects of the result should be studied. Also, the possible mechanisms which may cause the increased occurrence rate of the bursts during slow solar wind should be a topic of future studies.

Acknowledgements. The work of E. Kilpua and M. Myllys is funded by Academy of Finland projects 1218152 and 1267087.

The topical editor L. Blomberg thanks the two anonymous referees for help in evaluating this paper.

\section{References}

Akasofu, S.-I.: Energy coupling between the solar wind and the magnetosphere, Space Sci. Rev., 28, 121-190, 1981.

Angelopoulos, V., Baumjohann, W., Kennel, C. F., Coroniti, F. V., Kivelson, M. G., Pellat, R., Walker, R. J., Lühr, H., and Paschmann, G.: Bursty bulk flows in the inner central plasma sheet, J. Geophys. Res.-Space, 97, 4027-4039, 1992.

Angelopoulos, V., Kennel, C. F., Coroniti, F. V., Pellat, R., Kivelson, M. G., Walker, R. J., Russell, C. T., Spence, H. E., Baumjohann, W., and Feldman, W. C.: Characteristics of ion flow in the quiet state of the inner plasma sheet, Geophys. Res. Lett., 20, 17111714, 1993.

Angelopoulos, V., Kennel, C. F., Coroniti, F. V., Pellat, R., Kivelson, M. G., Walker, R. J., Russell, C. T., Baumjohann, W., Feldman, W. C., and Gosling, J. T.: Statistical characteristics of bursty bulk flow events, J. Geophys. Res.-Space, 99, 21257-21280, 1994.

Auster, H.U., Glassmeier, K.H., Magnes, W., Aydogar, O., Baumjohann, W., Constantinescu, D., Fischer, D., Fornacon, K. H., Georgescu, E., Harvey, P., Hillenmaier, O., Kroth, R., Ludlam, M., Narita, Y., Nakamura, R., Okrafka, K., Plaschke, F., Richter, I., Schwarzl, H., Stoll, B., Valavanoglou, A., and Wiedemann, M.: The THEMIS fluxgate magnetometer, The THEMIS Mission, Springer, New York, 235-264, 2009.
Baumjohann, W., Paschmann, G., and Lühr, H.: Characteristics of high-speed ion flows in the plasma sheet, J. Geophys. Res.Space, 95, 3801-3809, 1990.

Crooker, N. U., Feynman, J., and Gosling, J. T.: On the high correlation between long-term averages of solar wind speed and geomagnetic activity, J. Geophys. Res., 82, 1933-1937, 1977.

Dungey, J. W.: Interplanetary magnetic field and the auroral zones, Phys. Rev. Lett., 6, 47-48, 1961.

Finch, I. and Lockwood, M.: Solar wind-magnetosphere coupling functions on timescales of 1 day to 1 year, Ann. Geophys., 25, 495-506, doi:10.5194/angeo-25-495-2007, 2007.

Finch, I. D., Lockwood, M. L., and Rouillard, A. P.: Effects of solar wind magnetosphere coupling recorded at different geomagnetic latitudes: Separation of directly-driven and storage/release systems, Geophys. Res. Lett., 35, L21105, doi:10.1029/2008GL035399, 2008.

Juusola, L., Østgaard, N., Tanskanen, E., Partamies, N., and Snekvik, K.: Earthward plasma sheet flows during substorm phases, J. Geophys. Res.-Space, 116, A10228, doi:10.1029/2011JA016852, 2011a.

Juusola, L., Østgaard, N., and Tanskanen, E.: Statistics of plasma sheet convection, J. Geophys. Res.-Space, 116, A08201, doi:10.1029/2011JA016479, 2011b.

McFadden, J. P., Carson, C. W., Larson, D., Ludlam, M., Abiad, R., Elliott, B., Turin, P., Marckwordt, M., and Angelopous V.: The THEMIS ESA Plasma Instrument and In-flight Calibration, Space Sci. Rev., 141, 277-302, 2008.

Meng, C-I., Tsurutani, B., Kawasaki, K., and Akasofu, S.-I.: Crosscorrelation analysis of the $\mathrm{AE}$ index and the interplanetary magnetic field $B_{z}$ component, J. Geophys. Res., 78, 617-629, 1973.

Nagai, T., Fujimoto, M., Saito, Y., Machida, S., Terasawa, T., Nakamura, R., Yamamoto, T., Mukai, T., Nishida, A., and Kokubun, S.: Structure and dynamics of magnetic reconnection for substorm onsets with Geotail observations, J. Geophys. Res.-Space, 103, 4419-4440, 1998.

Nakamura, M., Paschmann, G.,Baumjohann, W., and Sckopke, N.: Ion distributions and flows near the neutral sheet, J. Geophys. Res., 96, 5631-5649, 1991.

Ohtani, S., Miyashita, Y., Singer, H., and Mukai, T.: Tailward flows with positive $B_{Z}$ in the near-Earth plasma sheet, J. Geophys. Res.-Space, 114, A06218, doi:10.1029/2009JA014159, 2009.

Perreault, P. and Akasofu, S. I.: A study of geomagnetic storms, Geophys. J. Int., 54, 547-573, 1978.

Pulkkinen, T. I., Partamies, N., Kissinger, J., McPherron, R. L., Glassmeier, K.-H., and Carlson, C.: Plasma sheet magnetic fields and flows during steady magnetospheric convection events, J. Geophys. Res.-Space, 118, 6136-6144, 2013.

Raj, A., Phan, T., Lin, R. P., and Angelopoulos, V.: Wind survey of high-speed bulk flows and field-aligned beams in the near-Earth plasma sheet, J. Geophys. Res., 107, 1419, 2002.

Shiokawa, K., Baumjohann, W., and Haerendel, G.: Braking of high-speed flows in the near-Earth tail, Geophys. Res. Lett., 24, 1179-1182, 1997.

Stamper, R., Lockwood, M., Wild, M. N., and Clark, T. D. G.: Solar causes of the long-term increase in geomagnetic activity, J. Geophys. Res.-Space, 104, 28325-28342, 1999.

Tsurutani, B. T. and Gonzalez, W. D.: The efficiency of "viscous interaction" between the solar wind and the magnetosphere during 
intense northward IMF events, Geophys. Res. Lett., 22, 663-666, 1995.

Wang, C.-P., Lyons, L., Weygand, J., Nagai, T., and McEntire, R.: Equatorial distributions of the plasma sheet ions, their electric and magnetic drifts, and magnetic fields under different interplanetary magnetic field $\mathrm{B}_{z}$ conditions, J. Geophys. Res.-Space, 111, A04215 doi:10.1029/2005JA011545, 2006.
Wiltberger, M., Pulkkinen, T. I., Lyon, J. G., and Goodrich, C. C.: MHD simulation of the magnetotail during the December 10, 1996, substorm, J. Geophys. Res.-Space, 105, 27649-27663, 2000. 